\title{
GEM 132
}

National Cancer Institute

\section{Source}

National Cancer Institute. GEM 132. NCI Thesaurus. Code C1790.

A 20-mer mixed backbone oligonucleotide exhibiting anti-viral activity. GEM-132 is comprised of a strand of synthetic DNA with a modified RNA at each end (2-Omethylribonucleosides at the two 3- and four 5-terminal nucleotides) which is complementary to the intron-exon boundary of the UL35 and UL37 pre-mRNA transcripts of human cytomegalovirus. ( $\mathrm{NCl})$ 